\title{
Étude sur les zodiaques décorés dans les édifices religieux de Bourgogne au Moyen Âge
}

Mémoire de Master 2 de l'université de Bourgogne, sous la direction de Daniel Russo, Dijon, 2010.

\section{Angélique Ferrand}

\section{(2) OpenEdition}

Journals

Édition électronique

URL : https://journals.openedition.org/cem/12092

DOI : $10.4000 /$ cem. 12092

ISSN : 1954-3093

Éditeur

Centre d'études médiévales Saint-Germain d'Auxerre

Édition imprimée

Pagination : 411-418

ISSN : $1623-5770$

Référence électronique

Angélique Ferrand, «Étude sur les zodiaques décorés dans les édifices religieux de Bourgogne au Moyen Âge », Bulletin du centre d'études médiévales d'Auxerre / BUCEMA [En ligne], 15 | 2011, mis en ligne le 25 août 2011, consulté le 22 septembre 2022. URL : http://journals.openedition.org/cem/ 12092 ; DOI : https://doi.org/10.4000/cem.12092

Ce document a été généré automatiquement le 22 septembre 2022.

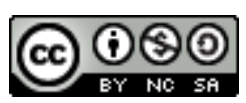

Creative Commons - Attribution - Pas d'Utilisation Commerciale - Partage dans les Mêmes Conditions 4.0 International - CC BY-NC-SA 4.0

https://creativecommons.org/licenses/by-nc-sa/4.0/ 


\section{Étude sur les zodiaques décorés dans les édifices religieux de Bourgogne au Moyen Âge}

Mémoire de Master 2 de l'université de Bourgogne, sous la direction de Daniel Russo, Dijon, 2010.

\section{Angélique Ferrand}

1 Le zodiaque, qui fait partie des thèmes figurés sur les portails de la basilique SainteMarie-Madeleine de Vézelay et de la cathédrale Saint-Lazare d'Autun, ne manque pas d'attirer l'attention et de susciter des interrogations. Ainsi, il était intéressant de centrer ce mémoire sur les zodiaques décorés dans les édifices religieux de Bourgogne $\mathrm{au} \mathrm{XII}{ }^{\mathrm{e}}$ siècle. Les zodiaques bourguignons comptent, en effet, parmi les exemples les plus importants de ce thème dans le panorama médiéval français, mais aussi occidental. Il était donc intéressant de présenter la tradition liée à ces images particulières et de comprendre leur résonance, afin de mettre en relief le corpus régional. Cependant, l'approche de ce thème iconographique nécessite d'aller au-delà des a priori sur ses origines astrologiques, qui ont souvent refréné les recherches. Étroitement liée au calendrier, mais conservant néanmoins son autonomie à l'intérieur des cycles concernés, l'iconographie zodiacale est issue d'une longue tradition, que nous avons tenté de saisir de diverses manières.

2 Sous la direction de Daniel Russo, ce travail avait pour point de départ la prise en compte des influences antiques sur le Moyen Âge, axe de recherche initié, notamment, par l'ouvrage de Jean Adhémar - Influences antiques dans l'art du Moyen Âge français (1939) ${ }^{1}$ - et par celui, qui lui fait presque écho, de Jean Seznec - La survivance des dieux antiques $(1940)^{2}$. Mis en regard dans notre introduction historiographique, ces deux ouvrages reflètent la vague warburgienne ayant trait à la question des origines de l'iconographie. Autre grand initiateur de ces questions, Émile Mâle (1862-1954), qui, au sein de son ouvrage consacré au XII ${ }^{\mathrm{e}}$ siècle ${ }^{3}$, souleva des questions fondamentales pour l'appréhension d'un thème complexe aux origines antiques. 


\section{Le corpus bourguignon : le zodiaque comme signes du temps}

3 Après un panorama des origines du zodiaque, de sa tradition iconographique et littéraire, et après l'avoir présenté comme révélateur de différentes ontologies et nombreuses manières d'être au monde, de le penser et de le représenter, nous avons établi un corpus bourguignon détaillé. Aux trois grands ensembles monumentaux, que sont Vézelay, Autun et Avallon, se sont ajoutés les vestiges de la mosaïque de pavement de Saint-Philibert de Tournus et des occurrences plus ponctuelles sur des chapiteaux ${ }^{4}$.

4 À Vézelay, Autun et Avallon, le zodiaque est présenté de manière similaire sur les voussures du portail, associé aux occupations des mois pour former un cycle calendaire. $\mathrm{Au}$ fil de l'étude, les zodiaques des voussures calendaires des portails sont apparus comme les acteurs d'une réflexion sur le temps et l'espace, constituant un cadre, une mise en scène de la théophanie. Les calendriers monumentaux bourguignons associent, en effet, les occupations des mois aux signes du zodiaque, placés dans des médaillons, selon une formule héritée de l'Antiquité, dans laquelle ces deux versants du calendrier réunissent les temps et les espaces. Les signes zodiacaux et les scènes des occupations des mois sont répartis de manière différente, mais réfléchie sur les voussures de ces portails.

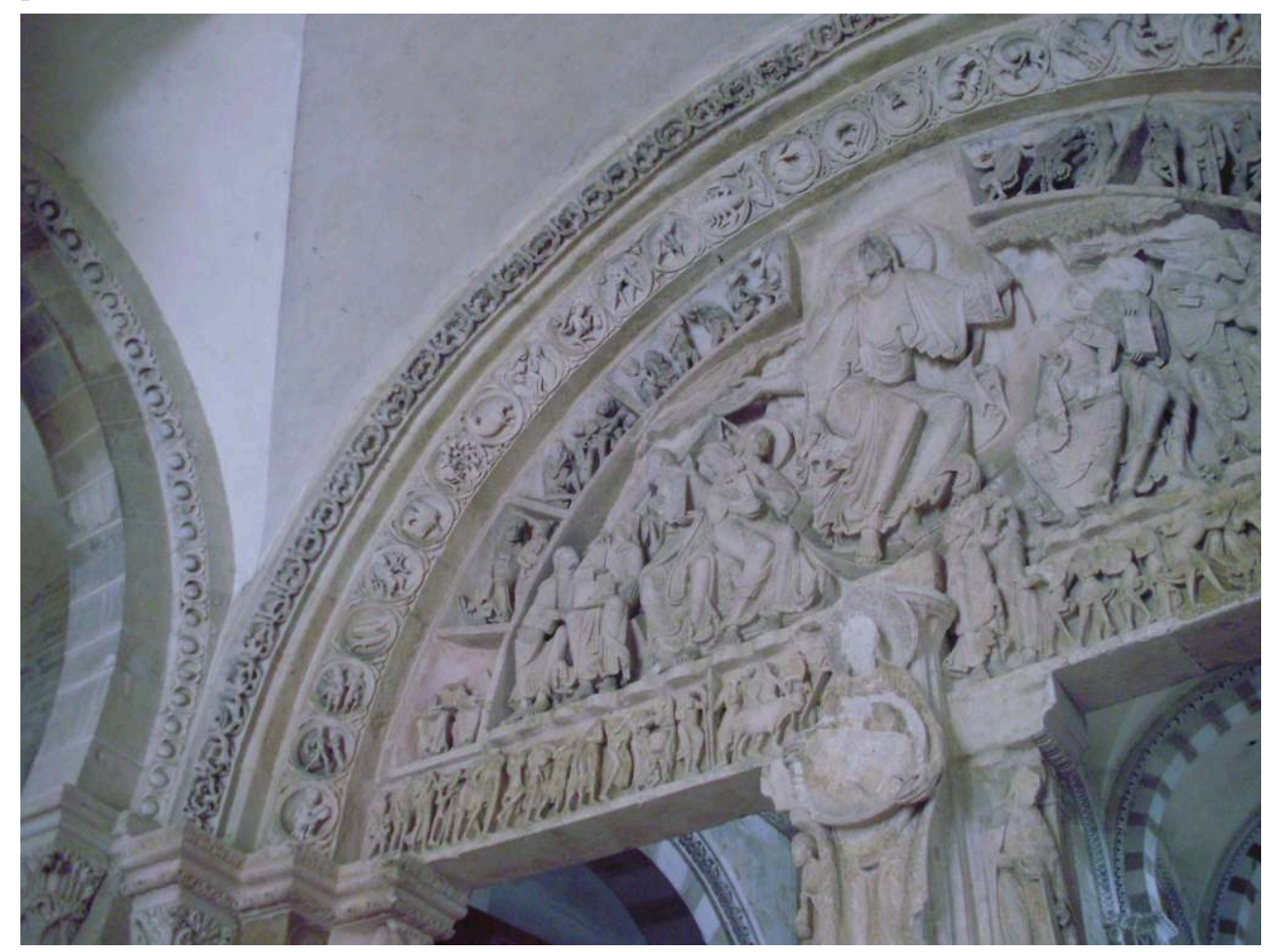

Fig. 1 - Vézelay, basilique Sainte-Marie-Madeleine, portail central (cl. A. Ferrand).

Dans le cas de Vézelay (fig. 1), dont le grand portail de l'avant-nef de l'église abbatiale a été réalisé entre 1120 et 1140, le zodiaque participe à l'illustration d'uneimago mundi et d'un ordo rerum régis par une pensée encyclopédique. L'archivolte est formée de deux voussures, l'une externe, végétalisée, la seconde accueillant des médaillons circulaires, dans lesquels sont figurées les occupations des mois alternant avec les signes du zodiaque. L'ensemble est ponctué de deux médaillons plus problématiques, pouvant 
être identifiés comme les Saisons et comme le Jour et la Nuit. Le sommet de l'archivolte est occupé par trois médaillons et un demi, chacun habités par un oiseau, un quadrupède, un acrobate et une sirène-poisson ${ }^{5}$. La voussure du calendrier ceint ainsi le tympan, sujet de tant de discussions, associant l'iconographie de la Pentecôte, de l'Ascension et de la mission évangélique donnée aux apôtres, avec pour point de jonction le Christ trônant dans une mandorle. La multiplicité "des temps» et des scènes, qui occupe ce portail, se résume par l'idée d'une "multiscène", autour de laquelle sont convoqués les signes du temps que sont les signes du zodiaque associés au rythme saisonnier des activités agricoles. La vision synthétique de plusieurs points de vue, mise en place autour du Christ, prône l'universalité du message évangélique donné à diffuser aux apôtres et illustre la quête du Salut par la connaissance et la compréhension du monde.

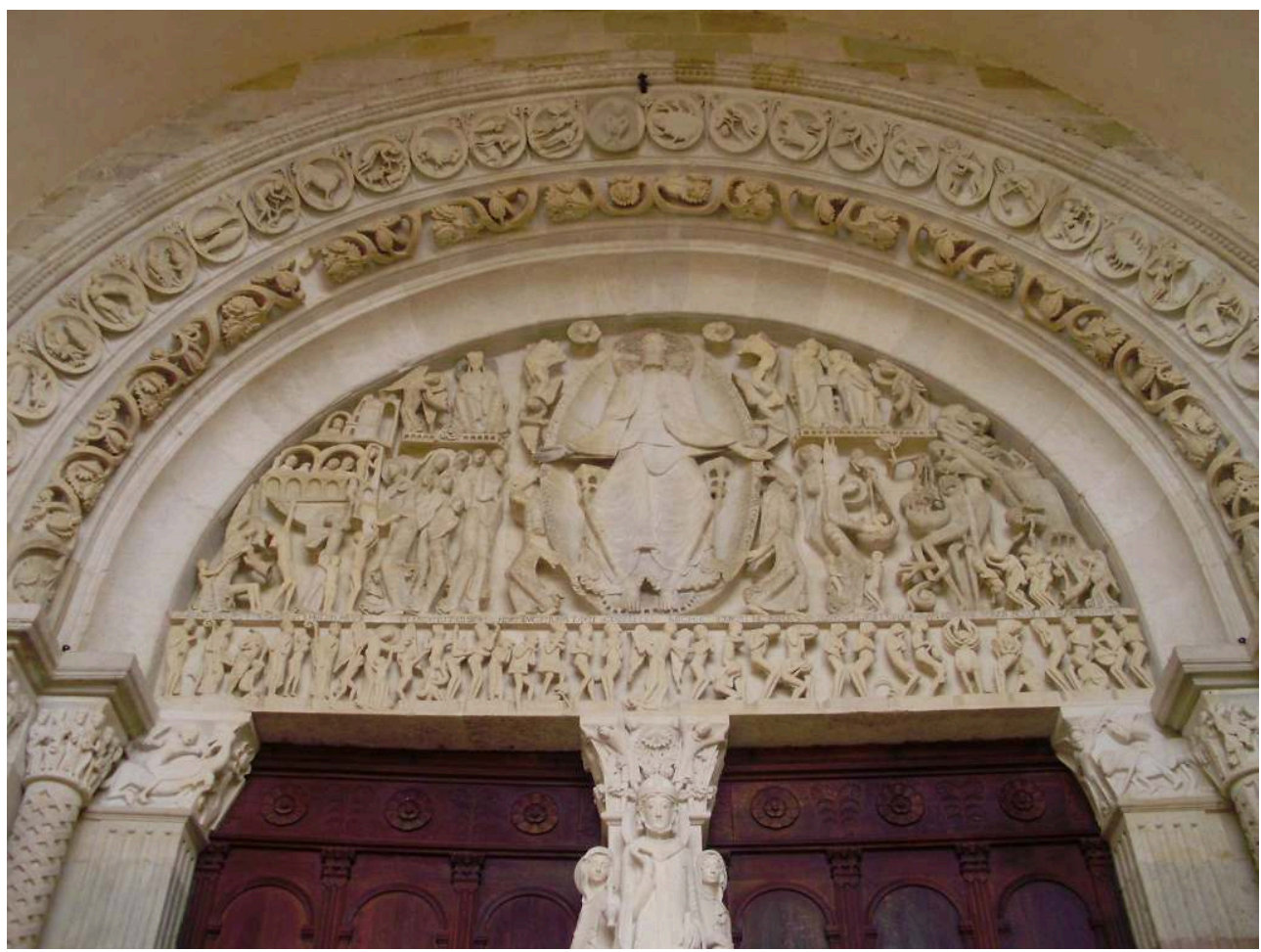

Fig. 2 - Autun, cathédrale Saint-Lazare, portail central (cl. A. Ferrand).

6 À Autun ${ }^{6}$ (fig. 2), dont le portail central est daté des environs de 1130-1140, le zodiaque, placé dans la voussure du calendrier, participe à la théophanie du Jugement dernier, qu'il situe spatialement et temporellement. De fait, cet ultime jugement s'inscrit universellement dans le temps et dans l'espace, qui se trouvent alors dépassés. Les procédés visuels, issus d'une ontologie analogique à visée encyclopédique, sont alors mis à profit pour servir l'illustration de la dimension universelle et la suprématie du Jugement dernier. La réunion sur la voussure des diverses unités cosmiques et temporelles, que représente l'association des occupations des mois, du zodiaque, des Saisons, à laquelle s'ajoute la figure centrale dite de l'Annus, préfigure le dépassement du temps par l'éternité du Salut. Cette dimension synthétique, que l'on trouve à la fois à Vézelay et à Autun, répond à un engouement pour la pensée encyclopédique, qui se développe au cours du XII ${ }^{e}$ siècle et qui profite, à cette période, de la « renaissance » du zodiaque comme vecteur d'un savoir hérité de l'Antiquité. 


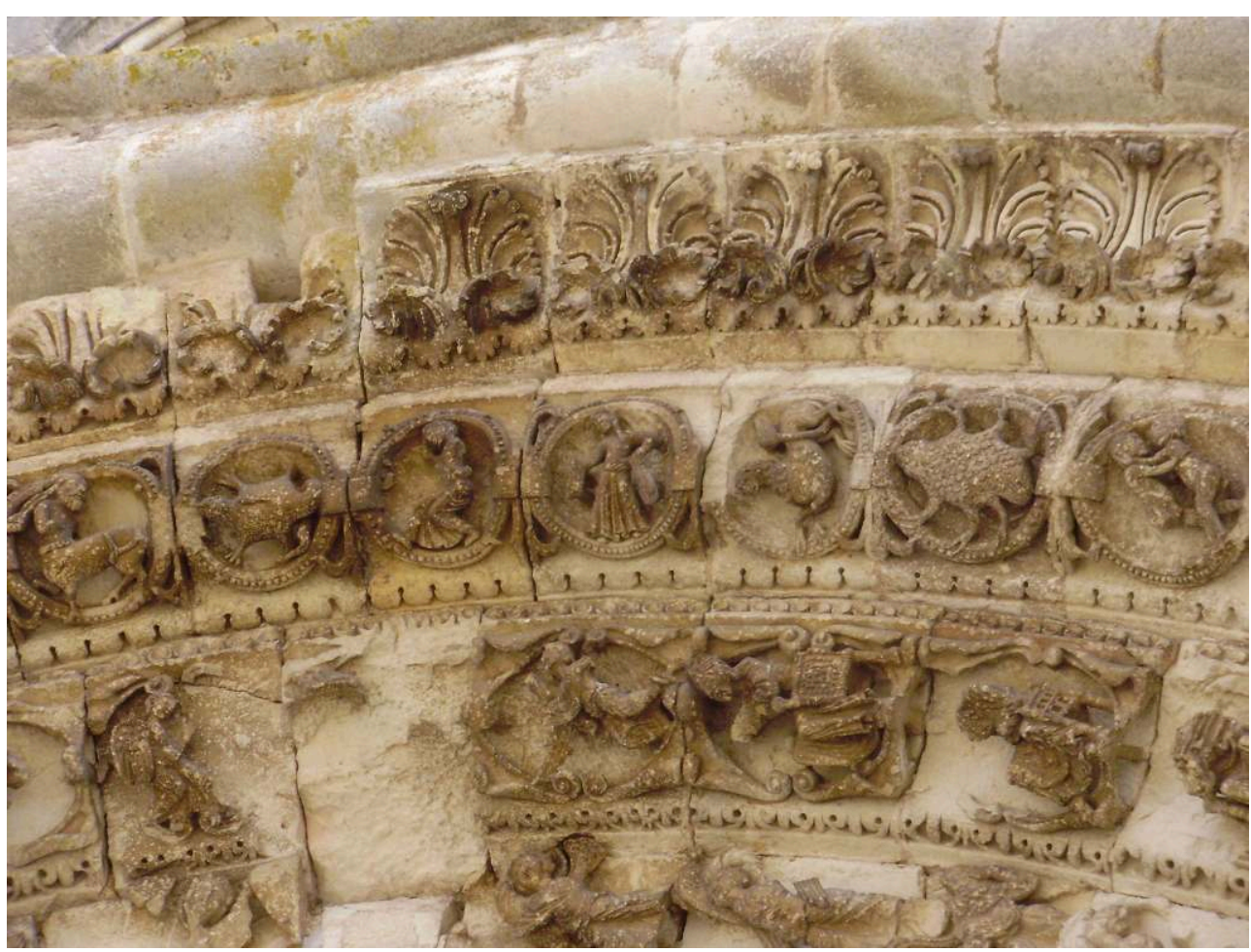

Fig. 3 - Avallon, Saint-Lazare, portail central, archivolte. Détail de la voussure du calendrier avec de gauche à droite : le Sagittaire, le Scorpion, la Balance, la Vierge, le Lion, le Cancer et les Gémeaux (cl. A. Ferrand).

7 Quant à Avallon (fig. 3), ce témoignage est intéressant, car le zodiaque est présent sur l'archivolte du portail, à une époque - réalisation située vers 1160-1170 - où le zodiaque a été, en quelque sorte, relégué aux parties basses. Ce portail trahit une certaine influence de Vézelay et d'Autun, mais correspond également à une évolution. Les occupations des mois ont été réparties sur le côté gauche, tandis que les signes du zodiaque occupent le reste de la voussure. L'ajout de figures supplémentaires sur le côté droit de cette voussure reste complexe à comprendre. Nous avons proposé d'identifier les sept dernières figures comme un second Sagittaire, la Lune ou la Nuit, un Vent soufflant vers la gauche, le Soleil ou le Jour, un Vent soufflant vers la gauche, le Verseau et, enfin, un Vent soufflant vers la droite. Le Sagittaire, doublé sur cette voussure, défend la particularité, mais aussi le succès de cette figure zodiacale, souvent difficile à distinguer du simple centaure. Dans ce portail, c'est donc la valeur temporelle du Salut qui semble être soulignée.

8 Ainsi, de manière générale, le zodiaque, allié aux occupations des mois, auxquels s'ajoutent des unités cosmologiques complémentaires, permet de faire valoir le temps terrestre et le « temps » de l'éternité, dans l'optique du Salut 7 .

\section{Le zodiaque dans un « monde enchevêtré »}

Par ailleurs, nous avons choisi de penser le zodiaque en termes de dynamique dans l'espace ecclésial. Il est, en effet, inscrit dans une tradition visuelle médiévale, établie par l'intermédiaire des enluminures de manuscrits liturgiques. Le zodiaque interagit avec l'espace qui l'entoure, qu'il s'agisse de l'espace écrit (manuscrit) ou construit (édifice). Déjà associé au Christ, notamment comme inscription spatio-temporelle de sa 
théophanie, le zodiaque fonctionne en partie comme repère visuel dans les manuscrits. Transposée sur la façade de l'église, la scène théophanique présentant le Christ entouré du zodiaque peut être reliée avec la porte évoquée dans la Bible, pour laquelle l'arc calendaire est une sorte d'arc de réunion entre des temps et des espaces. Par le biais d'une telle iconographie, l'idée d'un "monde enchevêtré » se trouve ainsi reflétée dans cette matérialisation de zones de contacts multiples. Dès lors, un jeu de relations s'instaure entre l'extérieur et l'intérieur, entre l'inférieur et le supérieur, entre le visible et l'invisible, dynamisant ainsi les rapports entre les différents pôles de l'espace ecclésial.

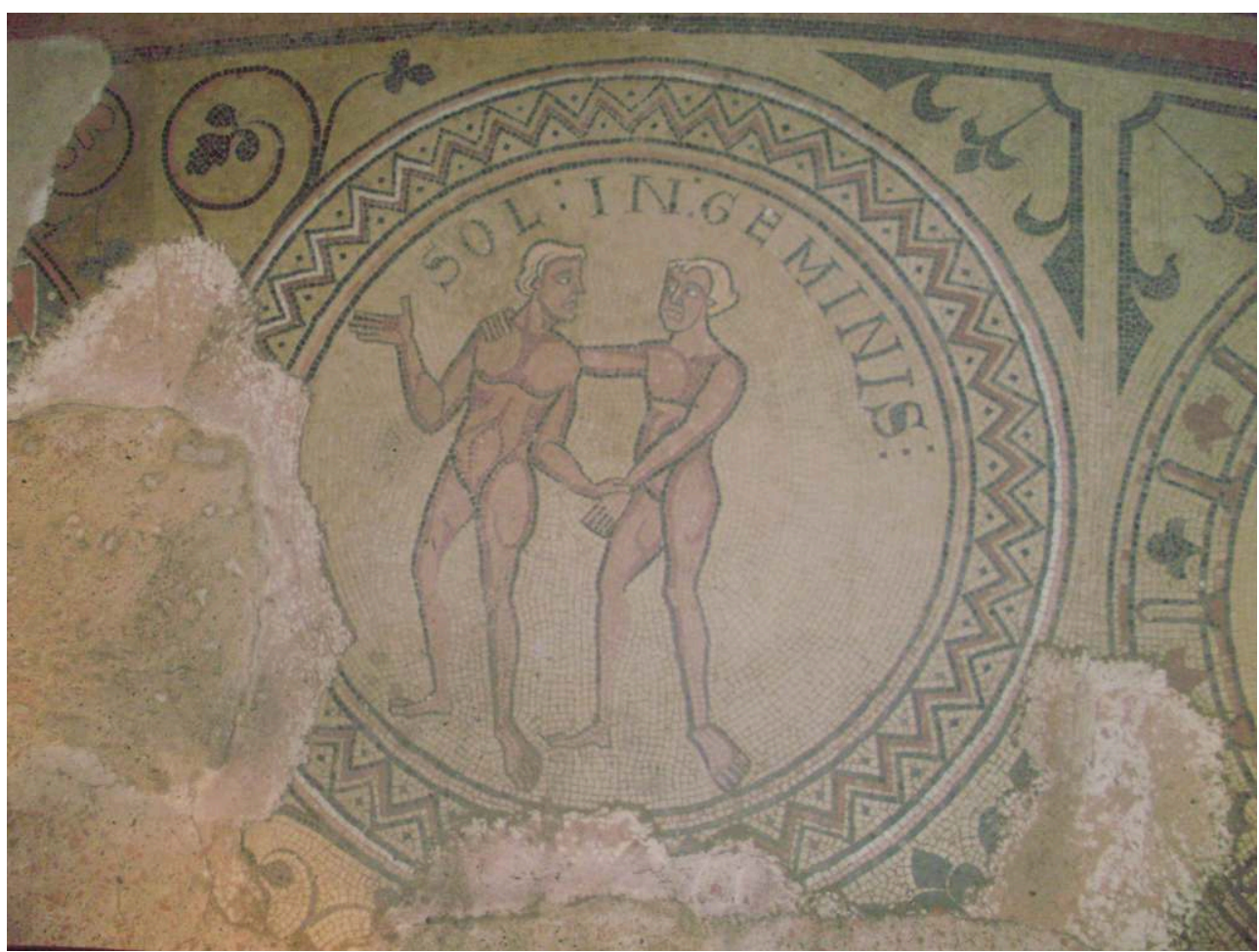

Fig. 4 - Tournus, Saint-Philibert, mosaïque de pavement du déambulatoire, v. 1200. Détail du médaillon du signe des Gémeaux (cl. A. Ferrand).

10 À Tournus (fig. 4), la mosaïque de pavement du déambulatoire présente un calendrier d'inspiration antique dans son organisation et le traitement de ses figures ${ }^{8}$. En circonscrivant le chœur, ce calendrier, qui est aujourd'hui très lacunaire, participe à la polarisation de l'édifice. Des médaillons circulaires accueillent les occupations des mois, alternant avec les signes du zodiaque. Fortement influencé par la tradition antique, ce pavement peut être mis en parallèle avec ses contemporains italiens, surtout ceux du nord de l'Italie. Un écho visuel semble pouvoir s'établir entre le cercle $\mathrm{du}$ zodiaque de la façade et celui du chœur, désignant ce dernier comme un espace d'accès au Salut. Par ailleurs, en Bourgogne, le zodiaque est adapté aux différents supports et espaces qui l'accueillent, permettant de structurer des dialogues entre eux et de faire écho aux formes concernées. Nous avons également pu relever des renvois entre le zodiaque et d'autres thématiques cosmologiques, qui s'établissent en partie grâce aux médaillons circulaires utilisés comme modules de présentation, aussi bien sur l'archivolte que sur les chapiteaux ou la mosaïque de pavement. Ces médaillons sont également le moyen de l'individualisation des signes du zodiaque sur l'archivolte 
ou dans la mosaïque et modélisent ces figures comme marqueurs de spatialité et de temporalité.

\section{Ontologies et modes de figuration : l'originalité bourguignonne}

11 La mise en regard des exemples bourguignons avec d'autres exemples français de la même époque (Poitou-Charentes) ou italiens fait ressortir une certaine originalité du «zodiaque bourguignon». Le mode de présentation des signes du zodiaque, notamment par le biais des médaillons circulaires, n'est pas aussi abouti ailleurs et le zodiaque est parfois subordonné aux occupations des mois. L'ordonnancement des signes et des scènes sur la courbe de la voussure facilite, en effet, une certaine mise en réseau visuelle, jouant en partie sur le principe de répétition.

La région Poitou-Charentes a en commun avec la Bourgogne de présenter une importante concentration d'occurrences zodiacales dans un cadre monumental. Des édifices, comme Saint-Pierre d'Aulnay ou Saint-Esprit de Fenioux, dont les portails furent réalisés au milieu du XII ${ }^{\mathrm{e}}$ siècle, présentent des cycles similaires. Le calendrier, formé des occupations des mois et des signes du zodiaque, se trouve, en effet, associé, sur les archivoltes des portails, avec l'Agneau porté en triomphe par des anges, le combat des vices et des vertus ainsi que la parabole des vierges sages et des vierges folles. Les occupations des mois et les signes zodiacaux, qui semblent subordonnés à celles-ci, sont répartis sur une voussure en fonction des claveaux, d'où l'expression d'«hommes-claveaux" utilisée par É. Vergnolle ${ }^{9}$. Ces cycles ont une visée eschatologique forte, mais le motif du calendrier est davantage repris comme un ensemble dans lequel la singularité du zodiaque s'efface. D'ailleurs, les erreurs iconographiques ponctuelles et le manque de détails dénoncent un manque de connaissance du sujet, le cycle du calendrier étant la reprise d'une formule. Ces cycles offrent également des sources d'hypothèses pour comprendre les voussures lacunaires du portail d'Avallon.

13 Pour le corpus italien, nous avons proposé un parallèle avec trois exemples du $\mathrm{XII}^{\mathrm{e}}$ siècle. L'ensemble des reliefs sculptés de la Porta dello Zodiaco de la Sacra di San Michele, près de Turin, datés des environs de 1122 et signés du maître Niccolò, traduisent une certaine connaissance de l'astronomie. La mosaïque de la crypte de l'église San Savino à Piacenza propose, quant à elle, une imago mundi, dans laquelle les médaillons circulaires, ceints d'inscriptions reprenant des vers d'Ausone (v. 309-v. 394), présentent les occupations des mois et les signes du zodiaque correspondant comme des marqueurs spatio-temporels. Enfin, pour la mosaïque de pavement de la cathédrale Santa Maria Assunta d'Otranto, réalisée vers 1163-1165, une narration prend forme au fil d'un arbre de vie, conçu comme un motif unificateur et classificateur, au sommet duquel sont portés les médaillons du calendrier mettant en valeur une réflexion eschatologique sur les temps et le Salut. Ainsi, les rapprochements avec d'autres exemples français et italiens font ressortir la dimension eschatologique des cycles auxquels participe le zodiaque.

14 D'autre part, afin d'observer l'évolution du zodiaque, tant du point de vue conceptuel qu'iconographique, il a paru intéressant d'enrichir cette approche d'un regard anthropologique. Les travaux, menés notamment sur les ontologies et sur les modes de 
figuration par Philippe Descola ${ }^{10}$, constituent une source de réflexions et une armature de concepts propices à l'observation des variations, dont le zodiaque fait l'objet en tant qu'élément cosmologique. Il s'agit d'appréhender les processus sous-jacents de la figuration, en mettant en rapport quatre ontologies clés, comme l'animisme, le totémisme, l'analogisme et le naturalisme, correspondant chacune à des modes figuratifs particuliers, ce qui explique l'idée d'ontologies "morphologisées ${ }^{11}$ » ou encore de "morphologie des relations ${ }^{12}$ ». De fait, de ces ontologies et différentes manières d'être au monde et de le penser, dépendraient donc des manières spécifiques de construire les images. À travers nos observations, l'ontologie analogique s'est bien distinguée comme fondamentale pour le zodiaque. Elle peut se résumer par l'idée de rayonnement, qui structure largement les dispositifs figuratifs accueillant celui-ci. Dans ces dispositifs circulaires ou à lecture cyclique, un processus d'homogénéisation externe d'éléments internes hétérogènes est, en effet, sensible. L'analogie fonctionne donc comme un moteur sémiologique et figuratif essentiel pour l'iconographie du zodiaque et de son « cercle des images».

Après ce mémoire, centré sur la Bourgogne, qui a fait apparaître des caractéristiques et a introduit certains processus de transmission, de reprise et de réappropriation, notre projet de recherches doctorales s'ouvrira sur un panorama spatial et chronologique destiné à mieux cerner l'évolution de l'iconographie du zodiaque. Cette approche sera formalisée autour de trois de ses concepts fondamentaux: le temps, l'espace et l'éternité, qui sont rendus sensibles par les images zodiacales des édifices cultuels, qu'ils soient païens à la fin de l'Antiquité, juifs pendant la période paléochrétienne ou chrétiens au cours du Moyen Âge ${ }^{13}$. Les manières de penser et de représenter le monde et le temps, selon les cultures et les périodes concernées, seront ainsi au cœur de nos réflexions sur le zodiaque.

\section{NOTES}

1. J. ADHÉMAR, Influences antiques dans l'art du Moyen Âge français, Londres, 1939 (réimp. Paris, 1996).

2. J. SEZNEC, La survivance des dieux antiques. Essai sur le rôle de la tradition mythologique dans l'humanisme et dans l'art de la Renaissance, Londres, 1940 (rééd. Paris, 1993).

3. É. MÂLE, L'art religieux du XII ${ }^{\mathrm{e}}$ siècle en France. Étude sur les origines de l'iconographie du Moyen Âge, Paris, 1922 (8e rééd., 1998).

4. Comme par exemple le chapiteau de Vézelay $n^{\circ} 19$, pile sud, avec la balance et les Gémeaux.

5. Au sujet de l'organisation de cette voussure et de l'identification problématique des figures, cf. V. FRANDON, «Du multiple à l'Un. Approche iconographique du calendrier et des saisons du portail de l'église abbatiale de Vézelay ", Gesta, 37/1 (1998), p. 74-87 ; P. DIEMER et D. DIEMER, « Le grand portail de Vézelay », in N. STRATFORD (dir.), Cluny, 910-2010. Onze siècles de rayonnement, Paris, 2010.

6. Cf. D. GRIVot et G. ZARNECKI, Gislebertus, sculpteur d'Autun, Paris, 1965 ( $2^{\mathrm{e}}$ éd.). 
7. Pour une réflexion sur le temps, cf. H. GŰNTHER, Le temps de l'histoire. Expérience du monde et catégories temporelles en philosophie de l'histoire de saint Augustin à Pétrarque, de Dante à Rousseau, Paris, 1995.

8. La mosaïque a été datée de l'époque suivant la campagne de reconstruction des années 1110-1140, période correspondant à l'apogée de l'abbaye, mais É. Vergnolle la date d'après le style des figures, soit vers 1200 , date qu'il nous semble devoir préférer. Cf. É. VERGNOLLE, « Sondages et fouilles archéologiques (1995 et 2001-2002). La redécouverte des mosaïques du déambulatoire ", in J. HENRIET, Saint-Philibert de Tournus. L'abbatiale du XI ${ }^{\mathrm{e}}$ siècle, Paris, 2008, p. 155-160. Pour ces questions de datation et le caractère antiquisant de cette mosaïque, voir L. GRODECKI, Le Moyen Âge retrouvé. De l'an Mil à l'an 1200, Paris, 1986, en part. « Le style des années 1200 ».

9. É. VERGNOLLE, L'art roman en France, Paris, 2003, p. 283.

10. P. Descola, Par-delà nature et culture, Paris, 2005 et P. DEscola (dir.), La fabrique des images. Visions du monde et formes de la représentation, Paris, 2010.

11. P. DescolA, « La fabrique des images », Anthropologie et société, 3 (2006), p. 167-182 [http:// id.erudit.org/iderudit/014932ar].

12. P. D escola, «L'Envers du visible: ontologie et iconologie», in Histoire de l'art et anthropologie, Paris, 2009, p. 12 [http://actesbranly.revues.org/181].

13. Thèse en cours, débutée en novembre 2010, sous la direction de Daniel Russo (université de Bourgogne) : Du zodiaque et des hommes. Temps, espace, éternité dans les édifices de culte, entre le $\mathrm{IV}^{\mathrm{e}}$ et le XII ${ }^{\mathrm{e}}$ siècle.

\section{INDEX}

Index géographique : France/Bourgogne

Mots-clés : édifice religieux, zodiaque 